\title{
A DISTRIBUTED VISION SYSTEM FOR BOAT TRAFFIC MONITORING IN THE VENICE GRAND CANAL
}

\author{
D.Bloisi, L.Iocchi, G.R.Leone, R.Pigliacampo \\ Dip. di Informatica e Sistemistica, University of Rome "La Sapienza", via Salaria 113, Rome, Italy \\ iocchi@dis.uniromal.it,leone@dis.uniromal.it \\ L.Tombolini \\ ECOTEMA-Ingegneria per l'Ambiente srl, Cannaregio 463, Venice, Italy \\ ltombolini@ecotema.it \\ L.Novelli \\ Archimedes, via Tagliamento 9, Rome, Italy \\ Inovelli@archimedes.it
}

\begin{abstract}
Keywords: Motion detection and Tracking, Distribuited surveillance, Boat traffic monitoring
Abstract: In this paper we describe a system for boat traffic monitoring that has been realized for analyzing and computing statistics of trafic in the Grand Canal in Venice. The system is based on a set of survey cells to monitor about $6 \mathrm{Km}$ of canal. Each survey cell contains three cameras oriented in three directions and covering about 250-300 meters of the canal. This paper presents the segmentation and tracking phases that are used to detect and track boats in the channel and experimental evaluation of the system showing the effectiveness of the approach in the required tasks.
\end{abstract}

\section{INTRODUCTION}

Wave motion has been recognized as one of the major causes of damage to the basement structures of historical buildings in Venice since the early 1960's, with the increase in boat mass and speed consequent to the diffusion of large diesel engines. Since then, the Venetian Municipal Authorities have been involved in defining rules and tradeoffs suitable for the need of mobility of goods, inhabitants and tourists on one hand, and the need of preservation of historical heritage on the other hand. Measures such as speed limits and strict traffic behavior rules, though, were proven to be only partly effective, due to the lack of continuous and autonomous traffic monitoring systems.

In the 1990's the public water-bus fleet was equipped with GPS satellite receivers and a first rough speed and trajectory monitoring system was put in place. In the years 2004-2005 a modern wide-range general fleet control system (SALOMON) was built up. The major feature of this system is the ability of the on-board equipment to define an extremely accurate boat position while holding a complete map of the city waterways with their related speed limits; in this way, the speed detected by the on-board Differential GPS receiver is immediately compared with the relevant speed limit and a signal is immediately issued to the boat driver in case of infringement. SALOMON system was proven to be very effective and precise; however, because of the need of a permanent installment of the intelligent navigation unit on the boats, its use is currently limited to the major resident fleets only.

In early 2006, the Municipal Administration launched the ARGOS project (Automatic Remote Grand Canal Observation System) for boat traffic monitoring, measurement and management along the Grand Canal of Venice based on automated vision techniques. This new system will answer to the specific requirements for the boat navigation rules in Venice while providing a combined unified view of the whole Grand Canal waterway. Such features far exceed the performance of any commercially available product. Therefore, a specific software has to be developed, based on the integration of advanced automated image analysis techniques. The ARGOS system will provide a unified man-machine interface both for the data detected through automatic vision and the ones detected by the former GPS-based systems. In turn, the precise GPS positioning data produced by the boats equipped with SALOMON navigation units will be used for $A R G O S$ cameras automatic geometric calibration.

In this paper we describe the implementation of the computer vision based system that allows for detecting and tracking the boats in the canal. After a brief overview of the ARGOS project given in Section 2 , we will describe Segmentation and Tracking processes in Sections 3 and 4. Section 5 describes some applications of the described modules and experimental evaluation of the system performance. 


\section{PROJECT OVERVIEW}

The ARGOS system is going to control a waterway of about $6 \mathrm{~km}$ length, 80 to 150 meters width, through some observation posts (Survey Cells). The system is based on the use of groups of IR/VIS cameras, installed just below the roof of several buildings leaning over the Grand Canal (a first prototype of a survey cell is visible in Figure 1). Detected images will be rectified and stitched together so as to generate a composite plain view, similar to a radar image. Each survey cell is composed of 3 optical sensors: one centre wide-angle (90 degree), orthogonal to the navigation axis, and two side deep-field cameras (50-60 degree). The resulting overall view field along the waterway could stretch over 250-300 meters end-to-end. The three cameras are connected to a local computer

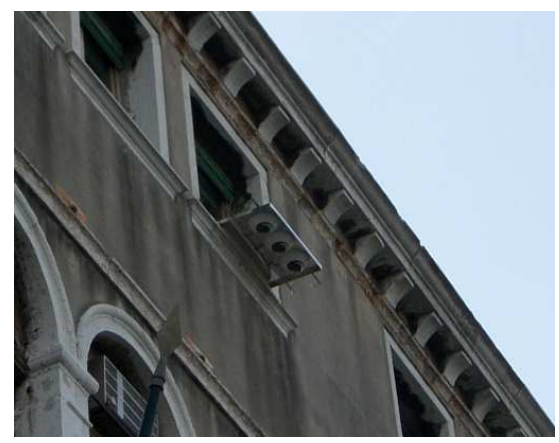

Figure 1: The survey cells are installed on the top of several buildings leaning over the Grand Canal

where images are processed through a two-level analysis software: a module for image re-sampling, rectification and stitching of contiguous images which, in turn, feeds a second software layer capable of detecting moving targets, delimiting them, finding their geometrical centre and filtering the time and position data by means of tracking algorithms to compute position, speed and direction of each target. The fields of view of each camera shall slightly overlap so as to allow the software to continuously follow and track the motion of each target through the whole composite view field of the survey cell, keeping each target labelled by a unique identifier (Track ID). Each survey cell is also equipped with a pan-tilt-zoom (PTZ) camera, for the automatic high-resolution tracking of selected targets. Due to the impossibility of wiring the survey cells together, all the system data traffic shall be conveyed through a private radio channel. As this channel has a limited bandwidth, most of the computation load shall be borne by the survey cells themselves. The system has to be designed for $24 / 7$ allweather day-night operation. The global system architecture is depicted in Figure 2.

The main ARGOS functions are: 1) Optical detection and tracking of moving targets present in the FOV; 2) Computing position, speed and heading of any moving target within the FOV of each camera;

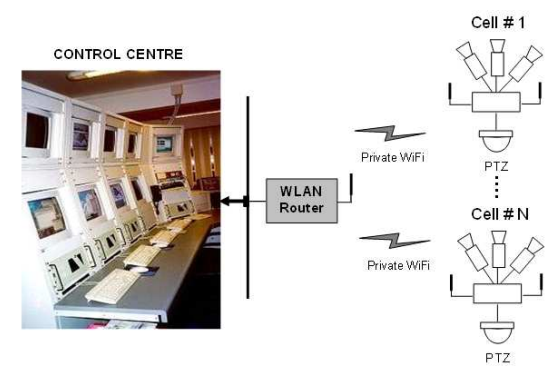

Figure 2: All the system data traffic shall be conveyed trough a private radio channel

3) Elaboration at survey cell level of any event (target appears, exits, stops, starts within the cells FOV) and transmission of any event to the Control Centre; 4) Connecting all the track segments related to the same target in the different cameras FOV into a unique trajectory and track ID; 5) Recording all the video frames together with the graphical information related to track IDs and trajectories; 6) Rectifying all the camera frames and stitching them into a composite plain image so as to show a plan view of the whole Grand Canal; 7) Allowing the operator to graphically select any target detected by the system and automatically activating the nearest PTZ camera to track the selected target.

In order to achieve ARGOS objectives we designed and developed a computer vision software which is a pipeline of image analysis and tracking techniques: we apply background subtraction and optical flow to the streams coming from the cameras to obtain observations (i.e., to detect the part of the image where the boats are); this information (position, dimension, versus, and velocity of a boat) is used by a set of Kalman Filters to track the targets over time. The next two sections explain our approach in details.

\section{SEGMENTATION}

Our goal is to detect the moving targets in the scenario (the boats): given a frame sequence from a fixed camera, we have to detect the zones of the images representing all the objects that are passing through. A common and effective technique to do that is called background subtraction. The background image is not fixed but must adapt to: gradual illumination changes and sudden ones (such as clouds), motion changes (camera oscillations), high frequency background objects (waves in our case), changes in the background geometry (such as parked boats). A simple way to build the background is to assign to a pixel the mean of all correspective ones in a set of images. But this method does not work well with illumination changes. Different methods for background modelling, updating and subtraction have been proposed. Two classification directions can be identified: how the background is modeled, 
how the model is updated. Statistical models have been widely used: either in the form of single Gaussians (Wren et al., 1997; Jabri et al., 2000) or mixture of Gaussians (Stauffer and Grimson, 2000; Elgammal et al., 2000), but also other models (median (Cucchiara et al., 2003), minimum-maximum values (Haritaoglu et al., 2000), etc.) proved effective. The choice of the model mostly depends on the kind of scenario in which the application runs.

In our case single Gaussian models are not adequate because we deal with outdoor environment with fluctuating background. This means that the distribution concerning a certain pixel often has more than one peak. So our approach is based on a mixture of Gaussians. The system computes the bar chart for every pixel (i.e., the approximation of the distribution) in the RGB color space and it clusters the raw data in sets based on distance in the color space. In order to save computational time, only the two bigger clusters are considered. If cardinality of the clusters are comparable, both the values are recorded, otherwise only the dominant one is considered. This solution allows for balancing efficiency with the need of representing fluctuations in the background.

When two boats are very near it is common to have an error called under segmentation due to the perspective of the camera view: the foreground image has only one bigger blob instead of two or more. To improve the detection in this situation we consider also the optical flow which correlates two consecutive frames. Every feature which is present in both the frames has a motion vector (direction, versus and value) which is not null if the position in the two frames is different. Optical flow is a good approximation of the motion over time in image coordinates. The system considers two directions for the central

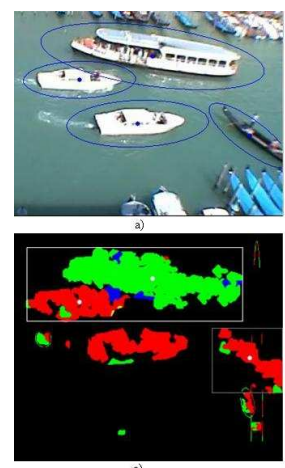

Figure 3: An example of under-segmentation: a) there are four boats in the scene but $b$ ) the system detects three blobs. c) Using optical flow the system understands the different directions of the boats and a) gives correct ellipses detections

camera (left to right and right to left) and four directions for the left and right cameras (because the perspective is not orthogonal) A typical example of under-segmentation correctly solved with optical flow is showed in Figure 3. Optical flow is often useful also when we have a single boat with a long wake on the back. Figure 4-b shows another typical segmentation error: the dimension of the boat is estimated more than double than the real one. Using optical flow (Figure 4-c) the system detects a big yellow blob that corresponds to the boat and other little ones in different directions which are the water waves. Also in this case optical flow provides a correct detection (Figure 4-d)

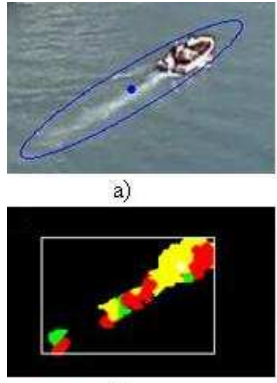

c)

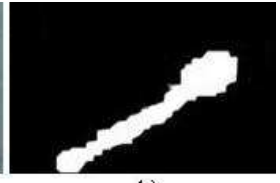

b)

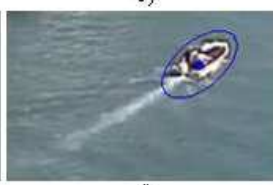

d)
Figure 4: Example of wrong segmentation caused by water waves: b) The blob in the foreground image is much bigger than the real boat and a) the system gives a wrong detection result but c) using optical flow the system distinguishes the boat from the waves and d) we obtain the correct detection

However, this approach fails when a boat is involved in particular maneuvers. For example, when a boat turns around itself, the optical flow may detect different directions for the different parts of the boat (e.g., one for the prow and another for the stern) and discard (for some frames) detection of such a target. Moreover, it is not useful when boats travel close by in the same direction. From an analysis of the performance of the segmentation process on many live video streams from our application scenario, we have evaluated that situations where optical flow worsen the performance of the segmentation process are very limited, with respect to the advantages in refining segmentation when two boats moving in different directions come close and in presence of long waves after the tracked boat. In summary, the algorithm used for image segmentation consists of the following steps: Background formation: a set $\mathrm{S}$ of $\mathrm{n}$ frames is used to build the background image B which represents only the static (i.e. non-moving) part of the scenario. This procedure is done continuously to adapt to the scenario's changes. Foreground computation: the difference between the current image I from the camera and the B image of the background gives the foreground image $\mathrm{F}$. This image is a binary image and contains only elements which are not in the background (new elements in the scenario); an example is Figure 3-b). Blob formation: the binary image B is analyzed in order to find connected components (i.e., blobs). Optical flow refining: for every detected blob optical flow is computed. If there is only one dominant moving direction (more than 70\%) the blob is 
confirmed, otherwise the blob is splitted in the different directions detected. In addition, optical flow is used to eliminate wave noise. Ellipse approximation: ellipses calculated on the size of the blobs represent an approximation of the boats detected. The centroids of the ellipses are used to track the boats over time (see Section 4).

\section{MULTI-HYPOTHESIS KALMAN FILTER TRACKING}

An optimal recursive data processing algorithm for tracking moving targets over time is the Kalman Filter (Welch and Bishop, 2004; Maybeck, 1979). Such a filter represents an efficient solution to the general problem of estimating the state of a discrete-time controlled process. When developing a multi-object tracking method, one usually has to deal with track initiation, track update including prediction and data association and track deletion. The process is divided into two fundamental steps: association: assignment of each incoming observation to a specific target track; estimate: the received observation is used to provide a state estimate of the associated track. Each time a new observation is received, it must be associated to the correct track among the set of the existing tracks, or, if it represents a new target, a new track must be created. Thus, the tracking system needs some mechanisms of Data Association and tracks management (see (Hall and Llinas, 2001)).

This is a single hypothesis approach which means that at all times an observation is associated to only one of the existing tracks. If a wrong association happens (i.e. an observation is associated to a wrong track) the system cannot recover from this error. In our case, especially when we have a very crowded scene, it is not straightforward to assign an observation to a certain track. For this reason we use a multi-hypothesis Kalman filter tracking system. Every time there is an observation that could be assigned to more than one track the system considers the two hypotheses for every candidate track (i.e. every track is divided in two new ones). This is called track split. This technique leads to the proliferation of the number of tracks and we need to detect and delete redundant track (track merging. In the next paragraph we illustrate with more details the single and multi hypothesis tracking phases.

\subsection{Single hypothesis tracking}

\subsubsection{Data Association}

The technique used for the data association is the Nearest Neighbor rule. When a new observation is received, all existing tracks are projected forward to the time of the new measurement. Then the observation is assigned to the nearest such estimate.

More generally, the distance calculation is computed to reflect the relative uncertainties (covariances) associated with each track and observation. The most widely used measure of the correlation between two mean and covariance pair $\left\{\mathbf{x}_{1}, \mathbf{P}_{1}\right\}$ and $\left\{\mathbf{x}_{2}, \mathbf{P}_{2}\right\}$, which are assumed to be Gaussian-distributed random variables, is:

$P_{\text {ass }}\left(\mathbf{x}_{1}, \mathbf{x}_{2}\right)=\frac{\exp \left(-\frac{1}{2}\left(\mathbf{x}_{1}-\mathbf{x}_{2}\right)\left(\mathbf{P}_{1}+\mathbf{P}_{2}\right)^{-1}\left(\mathbf{x}_{1}-\mathbf{x}_{2}\right)^{T}\right)}{\sqrt{2 \pi\left|\left(\mathbf{P}_{1}+\mathbf{P}_{2}\right)\right|}}$

If this quantity is above a given threshold, the two estimates are considered to be feasibly correlated. An observation is assigned to the track with which it has the highest association ranking. In this way, a multiple-target problem can be decomposed into a set of single-target problems.

\subsubsection{Track formation}

The nearest-neighbor rule is very simple and intuitive, but presents some difficulties. A first problem is in creating initial tracks for multiple targets, because some components of the vector state are not directly measurable. In the case of a single target, two observations can be accumulated to derive an estimate of such components. For multiple target, there is no obvious way to deduce such initial values: the first two observations could represent successive position of a single object or the initial detection of two distinct objects. Every subsequent observation could be the continuation of a known track or the start of a new one.

So when a new observation is obtained, if it is not highly correlated with an existing track, a new track is created and a new Kalman filter is initialized with the position $(x, y)$ observed and given to all the not observed components (e.g., velocity) a null value with a relatively high covariance. If the subsequent observations confirm the track existence, the filter will converge to the real state.

\subsubsection{Track deletion}

In many cases, some objects are not observed for a while, with the uncertainty in the state estimate increasing. Moreover the presence of noisy sensors can determine spurious observations, which give rise to spurious tracks. Thus, the tracking system needs an additional mechanism to recognize and delete tracks that do not receive any subsequent confirming observations.

We have considered, as indicative measure of the uncertainty in the state estimate of each target, the filter' $\mathrm{s}$ gain relative to the track:

$$
K_{t}=P_{t}^{-} H^{T}\left(H P_{t}^{-} H^{T}+R\right)^{-1}
$$

and experimentally established a threshold for the track deletion: if the received observations do not confirm a certain track for a period of time, the gain 
value grows exceeding the threshold and the track is deleted.

\subsection{Multi hypothesis tracking}

\subsubsection{Track splitting}

When two objects are sufficiently close together, the observations are highly correlated with more than one track. In these cases a misassignment can cause the Kalman-filtering process to converge very slowly, or fail to converge altogether. Moreover, tracks updated with misassigned observations (or not updated at all) will tend to correlate poorly with subsequent observations and may, therefore, be mistaken as spurious by the track deletion mechanism; mistakenly deleted tracks then necessitate subsequent track initiation and a possible repetition of the process.

The choice of a multi-hypothesis tracking has been made to give a solution to the problem of assignment ambiguity: when the correct association is not known, more association hypothesis are created. The new observation received is used to update all the tracks with which it has a probability association that exceeds the threshold value. A copy of the not updated track is also maintained (track splitting). Subsequent observations can be used to determine which assignment is correct.

\subsubsection{Track merging}

One important issue of the track splitting technique is a proliferation in the number of tracks. Because track splitting does not decompose multiple-target tracking into independent single-target problems, the deletion mechanism described in section 4.1.3 is not sufficient. For example, two hypothesis tracks may lock onto the trajectory of a single object; because both tracks are valid, the standard track-deletion mechanism cannot eliminate either of them.

The deletion procedure has to be modified to detect redundant tracks and, therefore, cannot look at just one track at a time. At each step, for each track the correlation with all the other tracks is calculated using equation (1). If the association probability between two tracks exceeds a threshold (experimentally established), one of the two tracks is deleted, keeping only the most significant hypothesis.

A successful example of this tracking method is shown in Figure 5: when two boats are very near they are detected as one (ellipse in Figure 5-a) because there is a foreground under segmentation error (Figure 5-b) and also the optical flow does not solve the problem because the boats go in the same direction(Figure 5-c). Thanks to the multi-hypothesis approach the system considers the wrong observation as a new track but it continues to track over time

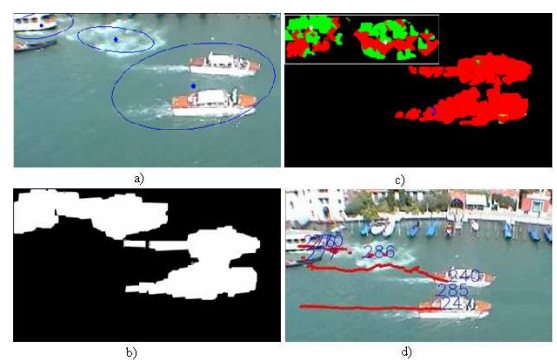

Figure 5: a)Two boats very near are detected as one because there is a b)Foreground under segmentation error and c)Optical flow does not solve the problem but d)With the multi-hypothesis method the system continues to track the boats separated over time

the former two because of the history of the observations(Figure 5-d).

\section{APPLICATION AND RESULTS}

In order to make available all the information gathered by the system in a useful way for the Venice $\mathrm{Mu}$ nicipal Authorities, we have developed different visualizations of the results of the system.

The main control window shows a live global view of the Grand Canal, integrating a GIS map with live information about position and velocity of the boats currently in the canal (see Figure 6). More specifically, we plot a colored dot in the current map location of each target with associated a few smaller dots representing its recent track. The color denotes the speed of the vehicle and other icons may appear close by to indicate specific events (such as, moving in a wrong way, stopping in a forbidden area, etc.) In addition, flow and density analysis are performed and displayed in order to have a global view of the traffic present in the canal at every time (example in Figure 7).

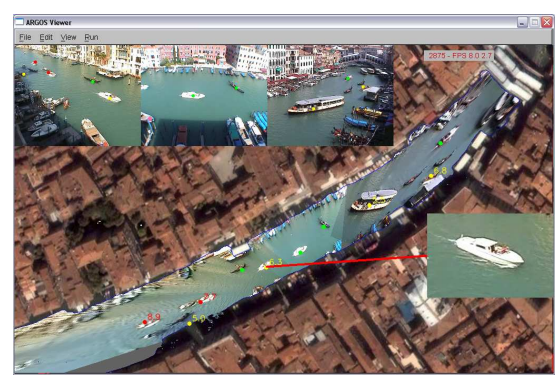

Figure 6: The main control window shows a live global view of the Grand Canal with live information about position and velocity of the boats currently in the canal.

The relevant information that must be extracted to achieve the functionalities described above can be divided in two groups: statistics measures and event detection.

The first kind of information is necessary to continuously monitor the traffic in the Canal. In particular, we want to calculate the traffic of boats moving in each direction for each survey-cell at different times 
of the day, as well as the boat density in the different areas of the Canal. To this end track analysis has been performed in order to compute the quantities of interest. For example, for computing the flow of boats passing within the area monitored by a survey cell we can define two virtual lines in the Canal and count the number of boats (i.e., tracks) passing these lines.

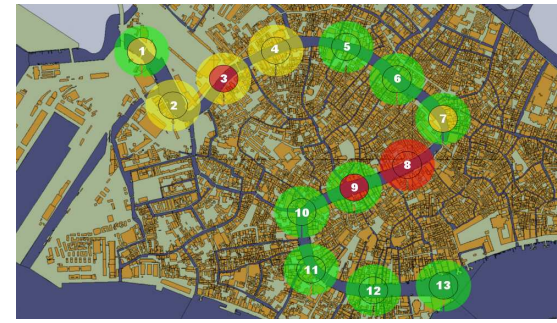

Figure 7: Density analysis are performed and displayed in order to have a global view of the traffic present in the canal at every time.

Another important measure is the velocity of the boats, since this is the main cause of wave motion that has been recognized as one of the major causes of damage to the basement structures of historical buildings in Venice. The velocity is computed by analysis of the world coordinates of the tracks: in particular to increase robustness to noise we compute the length of the path done in the last $n$ frames (e.g., $n=10$ ) as the sum of the distances between consecutive points and divide this value for the time duration of the path.

As for event detection, the main situations that must be automatically detected are: speed limits, i.e., detecting boats going at a velocity greater than a given threshold; parallel travel, i.e., detecting boats that move parallel and close each other for long time ${ }^{1}$; wrong directions, i.e., detecting boats moving in the wrong direction in one-way pieces of the Canal; forbidden stops, i.e., detecting boats stopping in forbidden areas.

Event detection is again based on specific analysis of the determined tracks. Speed limit is checked using two thresholds (that have been fixed to $5 \mathrm{Km} / \mathrm{h}$ and 7 $\mathrm{Km} / \mathrm{h}$ ) and each target is labeled with a color: green for speed below $5 \mathrm{Km} / \mathrm{h}$, yellow for speed between 5 and $7 \mathrm{Km} / \mathrm{h}$ and red for speed above $7 \mathrm{Km} / \mathrm{h}$. The visualization of colored dots in the GIS map makes it possible to quickly detect speed limit violations. Moreover, the system automatically records the tracks moving at a velocity above $7 \mathrm{Km} / \mathrm{h}$ for some time, allowing for subsequent analysis and post-processing. Parallel travel is detected by track analysis, in which we first detect parallel motion (by projecting the position of one boat to the direction line of the other) and then computing the distance between the two direction lines. A pair of parallel boats that maintain this distance below a given threshold for more than a given amount of time will generate an automatic alert.

\footnotetext{
${ }^{1}$ This is currently forbidden by Municipal Authorities.
}

Also in this case automatic recording will allow subsequent analysis. Finally, the other events are detected by defining zones in the canal that activate the corresponding checking procedures: for wrong direction we simply check that boats move in the right direction, for stops detection we monitor the time in which a boat remains within a limited area.

\subsection{Performance evaluation}

The performance results reported here refer to the capability of the system to provide accurate traffic statistics and event detection. It is important to notice that, although one goal of the project is to achieve best performance in associating a single track to each boat (i.e., avoiding track splitting) for its entire presence in the monitored area, statistics and events described above do not strictly require such a property. It is indeed sufficient that the boat is continuously tracked over short periods of time. Consequently, the following evaluation tests have been performed to measure the performance of the system in counting and shortterm tracking of passing boats.

All the tests have been executed with the same configuration of the software (i.e., with no specific optimization for a given functionality) on real images taken from a survey cell installed in Venice. The tests have been executed on long sequences of recorded videos that have been taken at the same frame rate of the software (about $5 \mathrm{fps}$ ).

Counting evaluation test. The first evaluation test has been set as follows. A virtual line has been put across the Canal in the field of view of a survey cell, the number of boats passing this line has been counted automatically by the system $\hat{n}$, and the same value is manually calculated by visually inspection $n$, the average percentage error is then computed as $\varepsilon=|\hat{n}-n| / n$. This error measures the general performance of the system, and it is useful to determine the general capabilities of the system of estimating traffic flow. However, this measure does not capture exactly all the errors made by the system. For example, if a boat is counted twice and another one is not detected, the error $\varepsilon$ is zero. An additional error measure is then calculated by considering the probability of making an error in counting a single boat passing the line

$$
P(e)=\frac{1}{n} \sum_{t=0}^{n} \delta\left(\hat{f}_{t}-f_{t}\right)
$$

where $\delta(\cdot)$ is 0 when the argument is 0 and 1 otherwise.

This experiment has been performed with three different videos of about 20 minutes each taken in different days. The results are shown in Table 1. 


\begin{tabular}{|c|c|c|c|c|c|}
\hline & duration & $n$ & $\hat{n}$ & $\varepsilon$ & $P(e)$ \\
\hline Video 1 & $32 \mathrm{~min}$ & 90 & 95 & 0.055 & 0.144 \\
\hline Video 2 & $30 \mathrm{~min}$ & 69 & 72 & 0.043 & 0.130 \\
\hline Video 3 & $25 \mathrm{~min}$ & 62 & 67 & 0.081 & 0.129 \\
\hline
\end{tabular}

Table 1: Counting errors.

Short-term tracking evaluation test . This test has been performed in order to evaluate the ability of the system to correctly perform short-term tracking, i.e. to maintain the same identifier to each target within a limited amount of time. To this end we have defined two passing lines in different places in the canal and measured the consistency of the tracks. More specifically, we denote with $n$ the number of boats passing through the two lines and with $c$ the number of boats that have the same id between the first and the second line. Thus $c / n$ represents the accuracy in short-term tracking. We repeated the experiment several times with different situations (single boat, two boats going in the same direction and two boats going in opposite direction). The results are shown in Table 2.

Table 2: Short-term tracking accurancy.

\begin{tabular}{|c|c|}
\hline & accuracy \\
\hline Single boat & 0.88 \\
\hline 2 boats same dir. & 0.75 \\
\hline 2 boats opp. dir. & 0.72 \\
\hline
\end{tabular}

In addition to the quantitative test above, we have performed qualitative tests. The live rectification of the perspective images in an integrated view (see Figure 6) allows to visually monitor all the area dedicated to a survey cell. The display of dots associated to tracked boats on top of the live rectified image allows for a comprehensive and augmented view that has proven to be very useful to the operators in the Municipal Control Center. Finally, automatic registration of events and image storage and retrieval facilities allows for subsequent analysis and for effective monitoring of the canal.

\section{CONCLUSION}

In this paper we have presented an implemented system for distributed monitoring and analysis of boat traffic in the Grand Canal of Venice. The system is mainly based on two processes: segmentation and tracking that computes tracks associated to the boats moving in the waterway. The characteristics of these processing modules are: robust and efficient segmentation that uses up to two Gaussians for modelling the background and optical flow to reduce segmentation errors; multi-hypothesis tracker based on Nearest Neighbors data association and Kalman filters.

The system has proven to be effective in performing the tasks of monitoring and trafic analysis, as reported by experimental results. Additional evaluation will be performed in order to provide additional expiremental evidence of the performance of the system as well as to improve its performance.

\section{ACKNOWLEDGEMENTS}

The project has been realized thanks to the view of the future and active participation of the City Council of Venice. In particular, special thanks to Lord Vice-Major of Venice, On. Michele Vianello, for his foresight in applying innovative technologies in the delicate and complex hystorical city as Venice. We are also grateful to the Responsible Manager Arch. Manuele Medoro and his staff for their constant support.

\section{REFERENCES}

Cucchiara, R., Grana, C., Piccardi, M., and Prati, A. (2003). Detecting moving objects, ghosts, and shadows in video streams. IEEE Transactions on Pattern Analysis and Machine Intelligence, 25(10):1337-1342.

Elgammal, A. M., Harwood, D., and Davis, L. S. (2000). Non-parametric model for background subtraction. In Proc. of the 6th European Conference on Computer $(E C C V)$, pages 751-767, London, UK. SpringerVerlag.

Hall, D. L. and Llinas, J. (2001). Handbook for multisensor data fusion (Electrical engineering and applied signal processing). CRC Press LLC.

Haritaoglu, I., Harwood, D., and Davis, L. S. (2000). W4: Real-time surveillance of people and their activities. IEEE Transactions on Pattern Analysis and Machine Intelligence, 22(8).

Jabri, S., Duric, Z., Wechsler, H., and Rosenfeld, A. (2000). Detection and location of people in video images using adaptive fusion of color and edge information. In Proc. of 15th International Conference on Pattern Recognition (ICPR'00), 4:4627.

Maybeck, P. S. (1979). Stochastic models, estimation and control. vol I. ACADEMIC PRESS.

Stauffer, C. and Grimson, W. E. L. (2000). Learning patterns of activity using real-time tracking. IEEE Trans. on Pattern Analysis and Machine Intelligence, 22(8):747-757.

Welch, G. and Bishop, G. (2004). An introduction to the kalman filter. Technical report, University of North Carolina at Chapel Hill, NC 27599-3175.

Wren, C. R., Azarbayejani, A., Darrell, T., and Pentland, A. (1997). Pfinder: Real-time tracking of the human body. IEEE Trans. on Pattern Analysis and Machine Intelligence, 19(7):780-785. 